Received by the editors: February 18, 2020; Accepted: October 26, 2020

\title{
A NEW LIFETIME DISTRIBUTION: TRANSMUTED EXPONENTIAL POWER DISTRIBUTION
}

\author{
Buğra SARACOĞLU and Caner TANIŞ \\ Department of Statistics, Selcuk University,Konya, TURKEY
}

\begin{abstract}
In this paper, we have introduced a new statistical distribution called as transmuted Exponential Power (TEP) distribution using the quadratic rank transmutation map proposed by Shaw and Buckley $25[26]$ in order to generate new distributions. We have also studied some statistical properties such as descriptive statistics (moments, variance, coefficient of skewness (CS) and kurtosis $(\mathrm{CK})$ ), point estimation (maximum likelihood estimation) and real data applications to illustrate usefulness of TEP distribution.
\end{abstract}

\section{INTRODUCTION}

In statistical literature, several lifetime distributions are introduced. Most of these distributions are generally obtained by compounding or mixing methodologies. In the other case, distributions are given through including an extra parameter to well-known distribution. By the way, family of distributions obtained using quadratic rank transmutation map (QRTM) proposed by Shaw and Buckley [25.26] is defined with cumulative distribution function (cdf) and probability density function (pdf)

$$
F(x)=(1+\lambda) G(x)-\lambda[G(x)]^{2}
$$

and

$$
f(x)=(1+\lambda) g(x)-2 \lambda G(x) g(x),
$$

respectively, where $G(x)$ denotes cdf of baseline distribution and $\lambda \in[-1,1]$ is transmuting parameter. If $\lambda=0$, cdf of the base distribution is obtained. In last decade, there are many studies about transmuted distributions in literature. For

2020 Mathematics Subject Classification. Primary 60E05; Secondary 62F10.

Keywords and phrases. Transmuted exponential power distribution, Quadratic rank transmutation map, Maximum likelihood estimation.

\bugrasarac@selcuk.edu.tr-Corresponding author; ctanis@selcuk.edu.tr

(D) 0000-0003-1713-2862; 0000-0003-0090-1661.

(C)2020 Ankara University Communications Faculty of Sciences University of Ankara-Series A1 Mathematics and Statistics 
example; Aryal and Tsokos [3.4] introduced transmuted Weibull and transmuted extreme value distributions. Ashour and Eltehiwy [5,6] proposed transmuted Lomax and transmuted exponentiated Lomax distributions, Merovci [18 21] introduced transmuted Rayleigh, transmuted Lindley, transmuted generalized Rayleigh and transmuted exponentiated Exponential distributions, Mahmoud and Mandouh 17 suggested transmuted Frechet distribution, Hussian 13 has introduced transmuted exponentiated Gamma distribution, Elbatal and Aryal [10] studied transmuted additive Weibull distribution, Khan et al. 14,16 introduced transmuted Weibull distribution, transmuted Kumaraswamy distribution and transmuted generalized Gompertz distribution, Shahzad and Asghar 24] proposed transmuted Dagum distribution, Al-Babtain et.al. 22 introduced the Kumaraswamy-transmuted exponentiated modified Weibull distribution.

Over two decades before, Smith and Bain [23] introduced the Exponential Power (EP) distribution by compounding exponential and Weibull distribution functions. The cdf and pdf of EP distribution are given

$$
G(x ; \alpha, \beta)=1-\exp \left[1-\exp \left(\left(\frac{x}{\alpha}\right)^{\beta}\right)\right]
$$

and

$$
g(x ; \alpha, \beta)=\frac{\beta}{\alpha}\left(\frac{x}{\alpha}\right)^{\beta-1} \exp \left(\left(\frac{x}{\alpha}\right)^{\beta}\right) \exp \left[1-\exp \left(\left(\frac{x}{\alpha}\right)^{\beta}\right)\right],
$$

respectively, where $\alpha>0, \beta>0$ and $x>0$. Many authors have focused on EP distribution recently. Some of these studies can be listed as follows; Chen 8], Barriga et al. 7], Akdam et al. 1].

The main purpose of this study is to suggest a new lifetime distribution as an alternative EP distribution by using QRTM. In Section 2, TEP distribution and its some statistical properties (moments, variance, CS, CK) are introduced. The maximum likelihood estimators (MLEs) for unknown parameters of introduced distribution are derived in Section 3. In Section 4, a Monte Carlo simulation study is performed to evaluate the performances of these estimators in terms of mean square errors (MSEs) and bias. In Section 5, two real data illustrations are given to show the applicability of this distribution in real life. In Section 6 , the conclusion remarks are given.

\section{Transmuted Exponential Power (TeP) Distribution}

Let $X$ be a random variable having TEP distribution with $\alpha, \beta$ and $\lambda$ parameters denoted by $\operatorname{TEP}(\alpha, \beta, \lambda)$. The cdf and pdf of this random variable are

$$
\begin{aligned}
F(x ; \alpha, \beta, \lambda)= & (1+\lambda)\left[1-\exp \left(1-\exp \left(\left(\frac{x}{\alpha}\right)^{\beta}\right)\right)\right] \\
& -\lambda\left[1-\exp \left(1-\exp \left(\left(\frac{x}{\alpha}\right)^{\beta}\right)\right)\right]^{2}
\end{aligned}
$$


and

$$
\begin{aligned}
f(x ; \alpha, \beta, \lambda)= & \frac{\beta}{\alpha}\left(\frac{x}{\alpha}\right)^{\beta-1} \exp \left(\left(\frac{x}{\alpha}\right)^{\beta}\right) \exp \left[1-\exp \left(\left(\frac{x}{\alpha}\right)^{\beta}\right)\right], \\
& \times\left[1+\lambda-2 \lambda\left(1-\exp \left(1-\exp \left(\left(\frac{x}{\alpha}\right)^{\beta}\right)\right)\right)\right],
\end{aligned}
$$

respectively. Where $-1 \leq \lambda \leq 1, \alpha, \beta>0$ and $x>0$. The cdf of EP distribution for $\lambda=0$ in Eq. (5) is derived. Figure 1 shows the possible shapes of the pdf of TEP distribution for various parameter values.
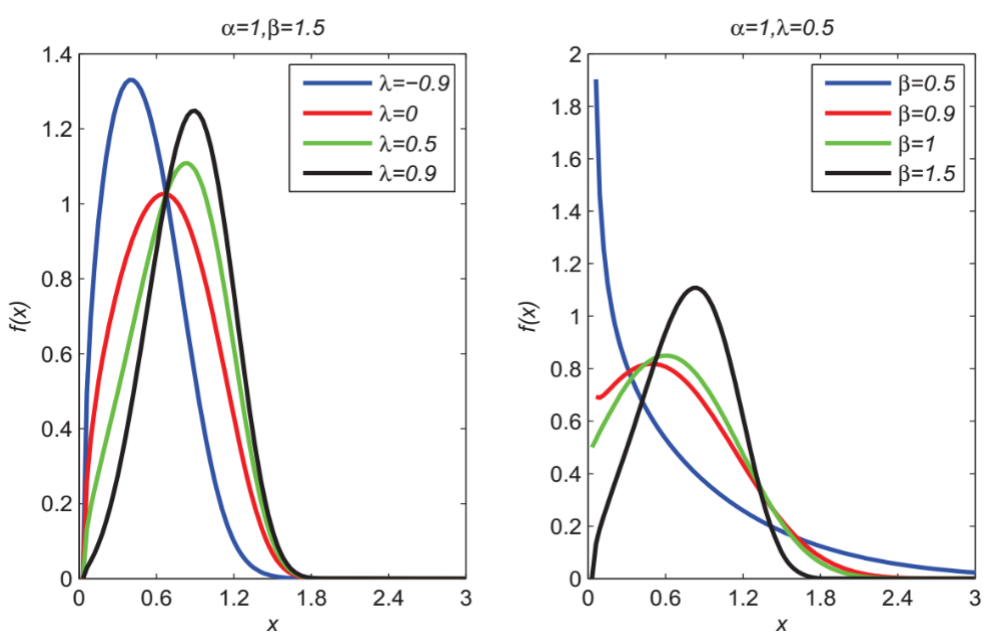

FIGURE 1. Plots of the TEP density function for various values of $\alpha, \beta$ and $\lambda$

The reliability function $(r f)$ and hazard function $(h f)$ of TEP distribution are defined as

$$
\begin{aligned}
R(t)= & 1-(1+\lambda)\left[1-\exp \left(1-\exp \left(\left(\frac{t}{\alpha}\right)^{\beta}\right)\right)\right] \\
& +\lambda\left[1-\exp \left(1-\exp \left(\left(\frac{t}{\alpha}\right)^{\beta}\right)\right)\right]^{2}
\end{aligned}
$$

and

$$
\begin{aligned}
h(t)= & \frac{\frac{\beta}{\alpha}\left(\frac{t}{\alpha}\right)^{\beta-1} \exp \left(\left(\frac{t}{\alpha}\right)^{\beta}\right) k(t, \alpha, \beta)}{1-(1+\lambda)[1-k(t, \alpha, \beta)]+\lambda[1-k(t, \alpha, \beta)]^{2}} \\
& \times[1+\lambda-2 \lambda(1-k(t, \alpha, \beta))],
\end{aligned}
$$

respectively. Where $k(t, \alpha, \beta)=\exp \left[1-\exp \left(\left(\frac{t}{\alpha}\right)^{\beta}\right)\right]$. Figure 2 shows that the possible shapes of $(h f)$ for TEP distribution at different parameter values. 

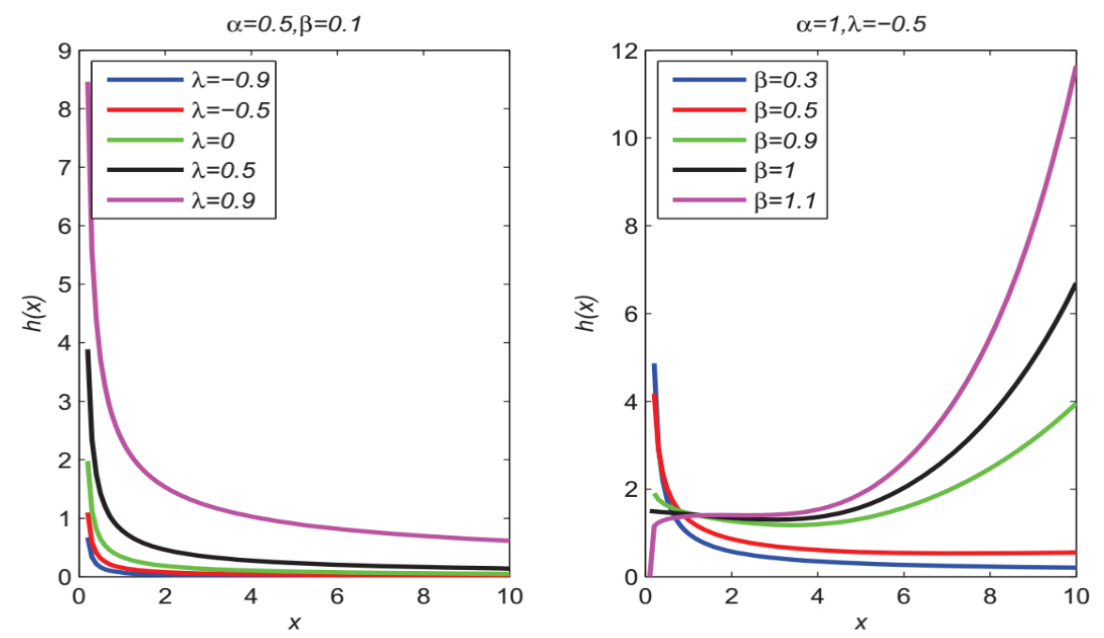

Figure 2. Plots of the TEP hazard function for various values of $\alpha, \beta$ and $\lambda$

2.1. Moments of TEP distribution. The rth moment of TEP distribution is

$$
\begin{aligned}
E\left(X^{r}\right) & =\int_{0}^{\infty} x^{r}((1+\lambda) g(x)-2 \lambda G(x) g(x)) d x \\
& =\left((1+\lambda) I_{1}-2 \lambda I_{2}\right)
\end{aligned}
$$

where $I_{1}$ and $I_{2}$ are

$$
\begin{aligned}
I_{1} & =\int_{0}^{\infty} x^{r} g(x) d x \\
& =\int_{0}^{\infty} x^{r} \frac{\beta}{\alpha}\left(\frac{x}{\alpha}\right)^{\beta-1} \exp \left(\left(\frac{x}{\alpha}\right)^{\beta}\right) s(x, \alpha, \beta) d x \\
& =\sum_{n=0}^{\infty} \frac{1}{n !} \alpha^{r} \int_{1}^{\infty}(\ln (u))^{n+(r / \beta)} \frac{e^{1-u}}{u} d u
\end{aligned}
$$

and

$$
\begin{aligned}
I_{2} & =\int_{0}^{\infty} x^{r} G(x) g(x) d x \\
& =\int_{0}^{\infty} x^{r}[1-s(x, \alpha, \beta)] \frac{\beta}{\alpha}\left(\frac{x}{\alpha}\right)^{\beta-1} \exp \left(\left(\frac{x}{\alpha}\right)^{\beta}\right) s(x, \alpha, \beta) d x \\
& =I_{1}-\sum_{n=0}^{\infty} \frac{1}{n !} \alpha^{r} \int_{1}^{\infty}(\ln (u))^{n+(r / \beta)} \frac{e^{2-2 u}}{u} d u,
\end{aligned}
$$


respectively. Where $s(x, \alpha, \beta)=\exp \left(1-\exp \left(\left(\frac{x}{\alpha}\right)^{\beta}\right)\right)$. Figure 3 illustrates the plots of some descriptive statistics such as expected value, variance, CS and CK at selected parameter values for TEP distribution.
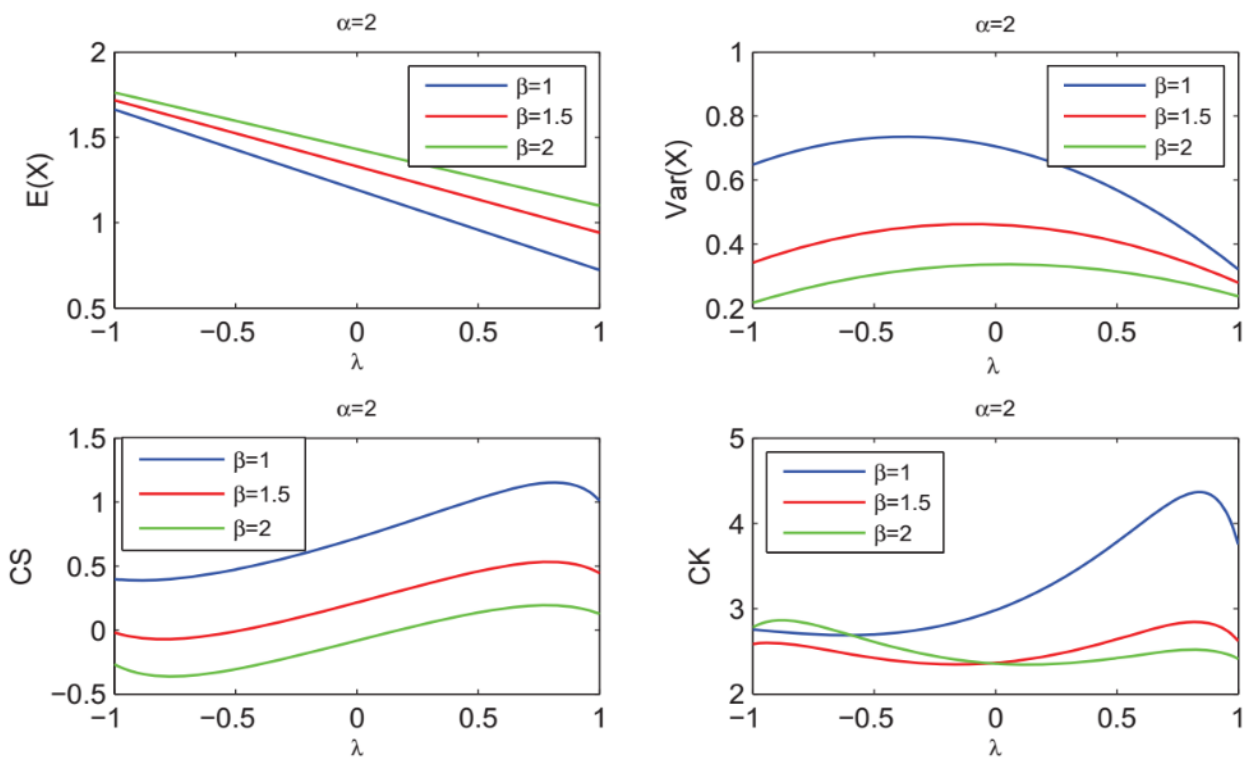

FiguRE 3. Descriptive statistics for $\alpha=2, \beta \in\{1,1.5,2\}, \lambda \in[-1,1]$.

2.2. Random Number Generator. The method of inversion transformation to generate random numbers from TEP distribution with parameters $\alpha, \beta$ and $\lambda$ is used as follows;

$$
F(x ; \alpha, \beta, \lambda)=(1+\lambda)[1-s(x, \alpha, \beta)]-\lambda[1-s(x, \alpha, \beta)]^{2}=u,
$$

where $s(x, \alpha, \beta)=\exp \left(1-\exp \left(\left(\frac{x}{\alpha}\right)^{\beta}\right)\right)$ and $u$ is a number generated from Uniform distribution shown as $U(0,1)$. Solution of Eq. 12 is given by

$$
x=\alpha\left[\ln \left(1-\ln \left(1-\left(\frac{1+\lambda-\sqrt{(\lambda+1)^{2}-4 \lambda u}}{2 \lambda}\right)\right)\right)\right]^{1 / \beta} .
$$

Maximum Likelihood Estimation 
Let $X_{1}, X_{2}, \ldots, X_{n}$ be a random sample having TEP distribution with parameters $\alpha, \beta$ and $\lambda$. Then the log-likelihood function is given by

$$
\begin{aligned}
\ell\left(\alpha, \beta, \lambda||_{-}\right)= & n \ln (\beta)-n \ln (\alpha)+\sum_{i=1}^{n}\left(\frac{x_{i}}{\alpha}\right)^{\beta}+n \\
& -\left(\sum_{i=1}^{n} \exp \left(\left(\frac{x_{i}}{\alpha}\right)^{\beta}\right)\right)+(\beta-1) \sum_{i=1}^{n} \ln \left(\frac{x_{i}}{\alpha}\right) \\
& +\sum_{i=1}^{n} \ln \left(\frac{x_{i}}{\alpha}\right) \ln \left(1+\lambda-2 \lambda\left(1-s\left(x_{i}, \alpha, \beta\right)\right)\right)
\end{aligned}
$$

Diferentiating the $\ell\left(\alpha, \beta, \lambda \mid \begin{array}{l}x \\ -\end{array}\right)$ with respect to $\alpha, \beta$ and $\lambda$ parameters, then equating to zero, non-linear equations is obtained as follows;

$$
\begin{aligned}
\frac{\partial \ell(\alpha, \beta, \lambda / \underline{x})}{\partial \alpha}=0 \Rightarrow & -\frac{n}{\alpha}-\sum_{i=1}^{n}\left(\frac{\beta}{\alpha}\right)\left(\frac{x_{i}}{\alpha}\right)^{\beta} \\
& -\sum_{i=1}^{n}\left(\frac{\beta}{\alpha}\right)\left(\frac{x_{i}}{\alpha}\right)^{\beta} \exp \left(\left(\frac{x_{i}}{\alpha}\right)^{\beta}\right)-\frac{n(\beta-1)}{\alpha} \\
+ & \sum_{i=1}^{n} 2 \lambda \frac{\beta}{\alpha}\left(\frac{x_{i}}{\alpha}\right)^{\beta} \exp \left(\left(\frac{x_{i}}{\alpha}\right)^{\beta}\right) \\
& \times s\left(x_{i}, \alpha, \beta\right)\left(1+\lambda-2 \lambda\left(1-s\left(x_{i}, \alpha, \beta\right)\right)\right)^{-1}=0 \\
\frac{\partial \ell(\alpha, \beta, \lambda / \underline{x})}{\partial \beta}=0 \Rightarrow & \frac{n}{\beta}+\sum_{i=1}^{n}\left(\frac{x_{i}}{\alpha}\right)^{\beta} \ln \left(\frac{x_{i}}{\alpha}\right) \\
& -\sum_{i=1}^{n}\left(\frac{x_{i}}{\alpha}\right)^{\beta} \ln \left(\frac{x_{i}}{\alpha}\right) \exp \left(\left(\frac{x_{i}}{\alpha}\right)^{\beta}\right) \\
& +\sum_{i=1}^{n} \ln \left(\frac{x_{i}}{\alpha}\right) \\
& -\sum_{i=1}^{n} 2 \lambda\left(\frac{x_{i}}{\alpha}\right)^{\beta} \ln \left(\frac{x_{i}}{\alpha}\right) \exp \left(\left(\frac{x_{i}}{\alpha}\right)^{\beta}\right) \\
& \times s\left(x_{i}, \alpha, \beta\right)\left(1+\lambda-2 \lambda\left(1-s\left(x_{i}, \alpha, \beta\right)\right)\right)^{-1}=0 \\
\frac{\partial \ell(\alpha, \beta, \lambda / \underline{x})}{\partial \lambda}=0 \Rightarrow-1+ & 2\left(1-\exp \left(\left(\frac{x_{i}}{\alpha}\right)^{\beta}\right)\right)\left(1+\lambda-2 \lambda\left(1-s\left(x_{i}, \alpha, \beta\right)\right)\right)^{-1}=0 .
\end{aligned}
$$

The MLEs of $\alpha, \beta$ and $\lambda$ are obtained by solving of Eqs. [15-17] via some numerical methods. 
Table 1. The MSEs and biases of $\alpha, \beta$ and $\lambda$.

\begin{tabular}{|c|c|c|c|c|c|c|c|}
\hline \multirow[b]{2}{*}{$\mathrm{n}$} & \multirow[b]{2}{*}{ parameter values } & \multicolumn{3}{|c|}{ bias } & \multicolumn{3}{|c|}{ MSE } \\
\hline & & $\hat{\alpha}$ & $\widehat{\beta}$ & $\hat{\lambda}$ & $\hat{\alpha}$ & $\hat{\beta}$ & $\hat{\lambda}$ \\
\hline 5 & & -0.4738 & 0.7255 & -0.8020 & 0.5023 & 1.1866 & 0.7368 \\
\hline 10 & & -0.3492 & 0.2620 & -0.6064 & 0.2502 & 0.1958 & 0.4855 \\
\hline 20 & & -0.3460 & 0.1159 & -0.4095 & 0.1858 & 0.0498 & 0.2452 \\
\hline 50 & $(2,0.8,0.1)$ & -0.3439 & 0.0578 & -0.2558 & 0.1510 & 0.0126 & 0.0796 \\
\hline 100 & & -0.3314 & 0.0433 & -0.2167 & 0.1320 & 0.0061 & 0.0532 \\
\hline 300 & & -0.2863 & 0.0275 & -0.1834 & 0.1052 & 0.0022 & 0.0428 \\
\hline 5 & & 0.0533 & 0.5609 & 0.6402 & 0.0274 & 0.7886 & 0.4485 \\
\hline 10 & & 0.0698 & 0.3201 & 0.5680 & 0.0195 & 0.2037 & 0.3830 \\
\hline 20 & & 0.0689 & 0.2132 & 0.4810 & 0.0138 & 0.0872 & 0.3277 \\
\hline 50 & $(0.4,0.6,-0.8)$ & 0.0582 & 0.1328 & 0.3541 & 0.0099 & 0.0398 & 0.2559 \\
\hline 100 & & 0.0424 & 0.0886 & 0.2503 & 0.0071 & 0.0241 & 0.1916 \\
\hline 300 & & 0.0198 & 0.0382 & 0.1148 & 0.0035 & 0.0101 & 0.0961 \\
\hline 5 & & -0.3532 & 0.7178 & -0.3549 & 0.3446 & 2.5125 & 0.2176 \\
\hline 10 & & -0.2487 & 0.2176 & -0.3094 & 0.1959 & 0.5131 & 0.2248 \\
\hline 20 & & -0.1759 & 0.0386 & -0.2589 & 0.1183 & 0.1932 & 0.2212 \\
\hline 50 & $(3,2,0.5)$ & -0.1123 & -0.0391 & -0.1976 & 0.0746 & 0.0882 & 0.1997 \\
\hline 100 & & -0.0832 & -0.0620 & -0.1645 & 0.0608 & 0.0610 & 0.1821 \\
\hline 300 & & -0.0273 & -0.0457 & -0.0754 & 0.0403 & 0.0308 & 0.1183 \\
\hline 5 & & -0.0513 & 0.1908 & -0.6024 & 0.0257 & 0.1503 & 0.4549 \\
\hline 10 & & -0.0563 & 0.0490 & -0.4850 & 0.0173 & 0.0248 & 0.3634 \\
\hline 20 & & -0.0671 & 0.0091 & -0.4005 & 0.0137 & 0.0080 & 0.3027 \\
\hline 50 & $(0.3,0.4,0.8)$ & -0.0630 & -0.0041 & -0.2968 & 0.0104 & 0.0031 & 0.2163 \\
\hline 100 & & -0.0516 & -0.0052 & -0.2235 & 0.0082 & 0.0017 & 0.1495 \\
\hline 300 & & -0.0310 & -0.0047 & -0.1314 & 0.0058 & 0.0006 & 0.0783 \\
\hline
\end{tabular}

\section{Simulation Study}

In this section, a Monte Carlo simulation study is performed to evaluate the performances of MLEs according to MSEs and biases. Algorithm steps regarding to simulation study are as follows;

Step 1. Random numbers are generated from TEP distribution with parameters $\alpha, \beta$ and $\lambda$ by using Eq. (13).

Step 2. MLEs of $\alpha, \beta$ and $\lambda$ are calculated by using Eqs. (15)-17) as based on 10000 replicates.

Step 3. The biases and MSEs of these estimators are simulated for different sample sizes as $5,10,20,50,100$ and 300 at selected parameter values $((\alpha, \beta, \lambda)=$ $(2,0.8,0.1),(0.4,0.6,-0.8)$,

$(3,2.0 .5)$, and $(0.3,0.4,0.8))$. The results of simulation study are presented in Table 1.

According to Table 1, it is clearly seen that the MSEs and biases of MLEs for all parameter cases decrease as sample sizes increases. This case indicates that estimate values approach to true values as sample size $n$ increases. 


\section{Real Data Analysis}

In this section, we aim to compare TEP distribution with other distributions in terms of goodness of fit measures to demonstrate the applicability of TEP distribution. Two real data sets have been used for these purposes. We have considered some goodness of fit measures such as the Akaike's Information Criterion (AIC), corrected Akaike's Information Criterion (AICc), $-2 \times \log$-likelihood value, Kolmogorov-Smirnov (KS) statistics and its p-value to compare the fits of the distributions for two data sets. These statistics are given as follows;

$$
\begin{gathered}
A I C=-2 \ell+2 k, \\
A I C c=A I C+\left(\frac{2 k(k+1)}{n-k-1}\right), \\
K S=\sup \left(\left|F(x)-F_{n}(x)\right|\right) .
\end{gathered}
$$

where $k$ is number of parameters, $n$ is sample size, $\ell$ is the value of log-likelihood function.

4.1. Operation and empirical data. The first real data set consist of 50 observations has been obtained by Dasgupta 9. This data set relates to holes operation on jobs made of iron sheet is given by in Table 2 .

Table 2. Operation and empirical data

\begin{tabular}{|l|l|l|l|l|l|l|l|l|l|}
\hline 0.04 & 0.02 & 0.06 & 0.12 & 0.14 & 0.08 & 0.22 & 0.12 & 0.08 & 0.26 \\
\hline 0.24 & 0.04 & 0.14 & 0.16 & 0.08 & 0.26 & 0.32 & 0.28 & 0.14 & 0.16 \\
\hline 0.24 & 0.22 & 0.12 & 0.18 & 0.24 & 0.32 & 0.16 & 0.14 & 0.08 & 0.16 \\
\hline 0.24 & 0.16 & 0.32 & 0.18 & 0.24 & 0.22 & 0.16 & 0.12 & 0.24 & 0.06 \\
\hline 0.02 & 0.18 & 0.22 & 0.14 & 0.06 & 0.04 & 0.14 & 0.26 & 0.18 & 0.16 \\
\hline
\end{tabular}

These data have been fitted to TEP, generalized Gompertz (GG) [11], transmuted generalized Gompertz (TGG) [16, transmuted Kumaraswamy (TKw) 14], transmuted Rayleigh (TR) [18, transmuted exponentiated exponential (TEE) [21] and transmuted Weibull (TW) 3 distributions. The density functions of the fitted 
Table 3. Parameter estimates(standard errors) for operation and empirical data

\begin{tabular}{cl}
\hline Distribution & \multicolumn{1}{c}{ MLEs } \\
\hline \multirow{2}{*}{ TEP } & $\hat{\alpha}=0.2400(0.0327), \hat{\beta}=1.5996(0.4081)$, \\
& $\hat{\lambda}=-0.0234(0.7000)$ \\
\hline \multirow{2}{*}{ TGG } & $\hat{a}=3.0808(3.2536), \hat{b}=7.5224(4.2694)$, \\
& $\hat{\alpha}=1.3521(0.5060), \hat{\lambda}=-0.1075(0.9151)$ \\
\hline \multirow{2}{*}{ GG } & $\hat{a}=2.5012(1.6916), \hat{b}=8.3737(3.0939)$, \\
& $\hat{\alpha}=1.2784(0.4692)$ \\
\hline \multirow{2}{*}{ TKw } & $\hat{a}=1.9335(0.3524), \hat{b}=30.1864(13.7921)$, \\
& $\hat{\lambda}=-0.2911(0.4408)$ \\
\hline TR & $\hat{\sigma}=0.1211(0.0110), \hat{\lambda}=-0.2645(0.3086)$ \\
\hline \multirow{2}{*}{ TW } & $\hat{\mu}=1.9917(0.3310), \hat{\sigma}=0.1708(0.0236)$, \\
& $\hat{\lambda}=-0.2722(0.4308)$ \\
\hline \multirow{2}{*}{ TEE } & $\hat{\theta}=2.6946(0.8398), \hat{\alpha}=12.4959(1.6663)$, \\
& $\hat{\lambda}=-0.5468(0.3189)$ \\
\hline
\end{tabular}

distributions are given by;

$$
\begin{aligned}
& T G G: f(x)=\alpha a e^{b x} e^{\left(-\frac{a}{b}\left(e^{(b x)-1}\right)\right)}\left[1-e^{\left(-\frac{a}{b}\left(e^{((b x)-1)}\right)\right)}\right]^{\alpha-1} \\
& \times\left[1+\lambda-2 \lambda\left(1-e^{\left(-\frac{a}{b} e^{((b x)-1)}\right)}\right)^{\alpha}\right], x>0, a, b, \alpha>0, \lambda \in[-1,1] \\
& G G: f(x)=\alpha a e^{b x} e^{\left(-\frac{a}{b}\left(e^{(b x)-1}\right)\right)}\left[1-e^{\left(-\frac{a}{b}\left(e^{((b x)-1)}\right)\right)}\right]^{\alpha-1}, x>0, a, b, \alpha>0 \\
& T E E: f(x)=\theta \alpha\left(1-e^{-\alpha x}\right)^{\theta-1} e^{-\alpha x}\left[1+\lambda-2 \lambda\left(1-e^{-\alpha x}\right)^{\theta}\right], x>0, a, \theta>0, \lambda \in[-1,1] \\
& T K w: f(x)=a b x^{a-1}\left(1-x^{a}\right)^{b-1}\left[1-\lambda+2 \lambda\left(1-x^{a}\right)^{b}\right], x \in[0,1], a, b>0,, \lambda \in[-1,1] \\
& T R: f(x)=\frac{x}{\sigma^{2}} e^{-\frac{x^{2}}{2 \sigma^{2}}}\left[1-\lambda+2 \lambda e^{-\frac{x^{2}}{2 \sigma^{2}}}\right], \quad x>0, \sigma>0, \lambda \in[-1,1] \\
& \mathrm{TW}: f(x)=\frac{\mu}{\sigma}\left(\frac{x}{\sigma}\right)^{\mu-1} e^{-\left(\frac{x}{\sigma}\right)^{\mu}}\left[1-\lambda+2 \lambda e^{-\left(\frac{x}{\sigma}\right)^{\mu}}\right], \quad x>0, \mu, \sigma>0, \lambda \in[-1,1]
\end{aligned}
$$

For operation and empirical data set, the MLEs (standard errors) of fitted distributions are given in Table 3 and the selection criteria statistics are given in Table 4. Furthermore, The plots which shows fits of distributions to this data set can be examined from Figure 4 and Figure 5.

4.2. Breaking Stress data. The second data set is with regard to breaking stress of carbon fibers of $50 \mathrm{~mm}$ length $(\mathrm{GPa})$ obtained by Nichols and Padgett 22$]$. This 
Table 4. Selection criteria statistics for operation and empirical data

\begin{tabular}{cccccc}
\hline Distribution & $-2 \log$ & AIC & AICc & K-S & p-value \\
\hline TEP & $\mathbf{- 1 1 4 . 9 4 0 1}$ & $\mathbf{- 1 0 8 . 9 4 0 1}$ & $\mathbf{- 1 0 8 . 4 1 8 4}$ & $\mathbf{0 . 0 8 9 3}$ & $\mathbf{0 . 8 1 9 9}$ \\
TGG & -114.5400 & -106.5400 & -105.6520 & 0.0898 & 0.8152 \\
GG & -114.5924 & -108.5924 & -108.0707 & 0.0936 & 0.7736 \\
TKw & -112.5020 & -106.5020 & -105.9800 & 0.1052 & 0.6375 \\
TR & -112.1173 & -108.1173 & -107.8620 & 0.1070 & 0.6162 \\
TW & -112.1179 & -106.1179 & -105.5962 & 0.1070 & 0.6163 \\
TEE & -106.5607 & -100.5607 & -100.0389 & 0.1462 & 0.2357 \\
\hline
\end{tabular}

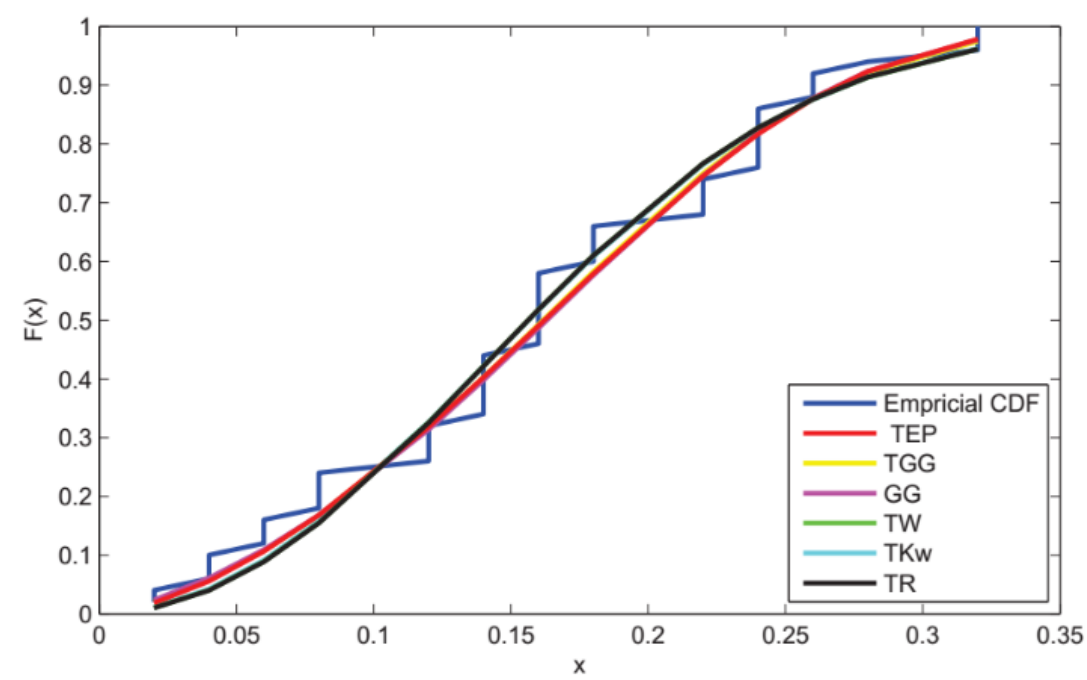

Figure 4. Empirical cdf and fitted cdfs for operation and empirical data set

data set consist of 66 observations has been used by Yousof et.al. 27]. The breaking stress data are presented in Table 5 .

Table 5. Breaking stress data

\begin{tabular}{|l|l|l|l|l|l|l|l|l|l|l|}
\hline 0.39 & 0.85 & 1.08 & 1.25 & 1.47 & 1.57 & 1.61 & 1.61 & 1.69 & 1.80 & 1.84 \\
\hline 1.87 & 1.89 & 2.03 & 2.03 & 2.05 & 2.12 & 2.35 & 2.41 & 2.43 & 2.48 & 2.50 \\
\hline 2.53 & 2.55 & 2.55 & 2.56 & 2.59 & 2.67 & 2.73 & 2.74 & 2.79 & 2.81 & 2.82 \\
\hline 2.85 & 2.87 & 2.88 & 2.93 & 2.95 & 2.96 & 2.97 & 3.09 & 3.11 & 3.11 & 3.15 \\
\hline 3.15 & 3.19 & 3.22 & 3.22 & 3.27 & 3.28 & 3.31 & 3.31 & 3.33 & 3.39 & 3.39 \\
\hline 3.56 & 3.60 & 3.65 & 3.68 & 3.70 & 3.75 & 4.20 & 4.38 & 4.42 & 4.70 & 4.90 \\
\hline
\end{tabular}

This data set has been fitted to TEP, exponential power (EP) 23], exponentiated exponential (EE) 12], transmuted exponentiated exponential (TEE) 21] and 


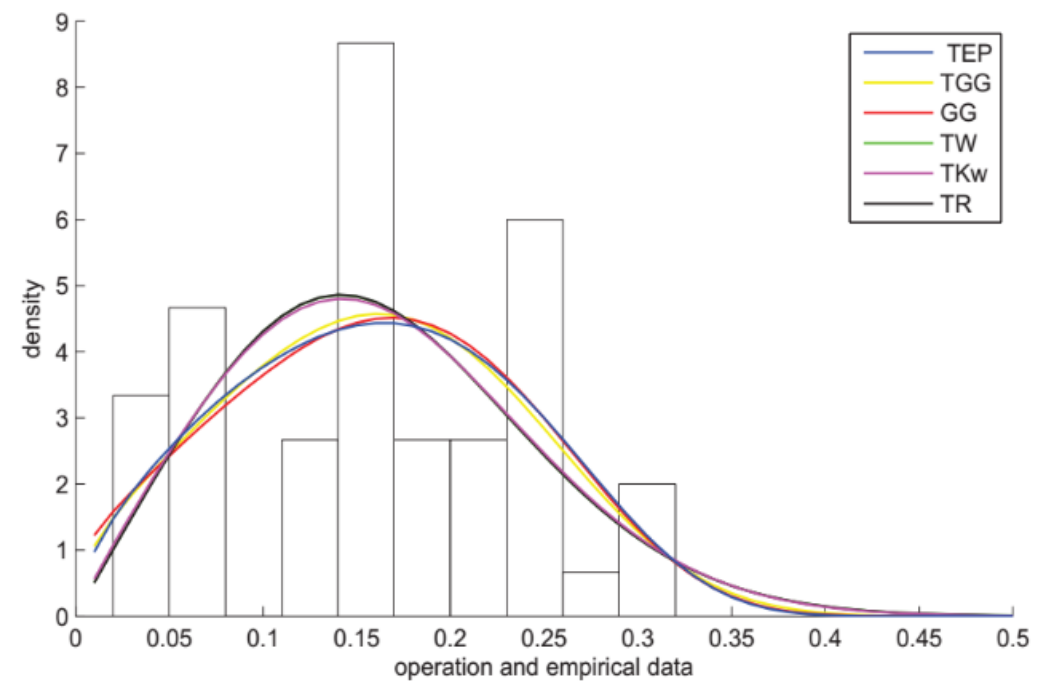

FIGURE 5. Fitted pdfs for operation and empirical data set

transmuted Rayleigh (TR) 18 distributions. The density functions of the fitted distributions are given by;

$$
\begin{aligned}
& E P: f(x)=\frac{\beta}{\alpha}\left(\frac{x}{\alpha}\right)^{\beta-1} \exp \left(\left(\frac{x}{\alpha}\right)^{\beta}\right) \exp \left[1-\exp \left(\left(\frac{x}{\alpha}\right)^{\beta}\right)\right], \quad x>0, \alpha, \beta>0 \\
& \begin{aligned}
& E E: f(x)= \theta \alpha\left(1-e^{-\alpha x}\right)^{\theta-1} e^{-\alpha x}, x>0, \alpha, \theta>0 \\
& T R: f(x)=\frac{x}{\sigma^{2}} e^{-\frac{x^{2}}{2 \sigma^{2}}}\left[1-\lambda+2 \lambda e^{-\frac{x^{2}}{2 \sigma^{2}}}\right], \quad x>0, \sigma>0, \lambda \in[-1,1] \\
& T E E: f(x)= \theta \alpha\left(1-e^{-\alpha x}\right)^{\theta-1} \\
& \quad \times e^{-\alpha x}\left[1+\lambda-2 \lambda\left(1-e^{-\alpha x}\right)^{\theta}\right], \quad x>0, \alpha, \theta>0, \lambda \in[-1,1]
\end{aligned}
\end{aligned}
$$

The MLEs of unknown parameters for these distributions and their standard errors are shown in Table 6 .

For breaking stress data, the comparison statistics of fitted distributions are given in Table 7. Also, the goodness of fit plots based on the empirical and theoretical cdfs and pdfs of fitted distributions can be seen from Figure 6 and Figure 7.

Table 7. Selection criteria statistics for breaking stress data 
Table 6. Parameter estimates(standard errors) for breaking stress data

\begin{tabular}{cl}
\hline Distribution & \multicolumn{1}{c}{ MLEs } \\
\hline \multirow{2}{*}{ TEP } & $\begin{array}{l}\hat{\alpha}=4.0683(0.2181), \hat{\beta}=2.8374(0.2716), \\
\hat{\lambda}=0.7487(0.2503)\end{array}$ \\
\hline EP & $\hat{\alpha}=3.6807(0.1182), \hat{\beta}=2.3799(0.2304)$ \\
\hline EE & $\hat{\theta}=9.1992(2.1491), \hat{\alpha}=1.0076(0.1002)$ \\
\hline \multirow{2}{*}{ TEE } & $\hat{\theta}=7.4605(2.1903), \hat{\alpha}=1.1195(0.1089)$, \\
& $\hat{\lambda}=-0.7773(0.1812)$ \\
\hline TR & $\hat{\sigma}=1.6957(0.0825), \hat{\lambda}=-0.9587(0.0930)$ \\
\hline
\end{tabular}

\begin{tabular}{cccccc}
\hline Distribution & $-2 \log$ & AIC & AICc & K-S & p-value \\
\hline TEP & $\mathbf{1 7 2 . 4 5 7 7}$ & $\mathbf{1 7 8 . 4 5 7 7}$ & $\mathbf{1 7 8 . 8 4 4 8}$ & $\mathbf{0 . 0 9 1 3}$ & $\mathbf{0 . 6 4 0 8}$ \\
EP & 174.7949 & 178.7949 & 178.9854 & 0.1126 & 0.3724 \\
EE & 190.7447 & 194.7447 & 194.9352 & 0.1550 & 0.0840 \\
TEE & 185.0412 & 191.0412 & 191.4283 & 0.1344 & 0.1844 \\
TR & 177.7488 & 183.7488 & 184.1359 & 0.1410 & 0.1447 \\
\hline
\end{tabular}

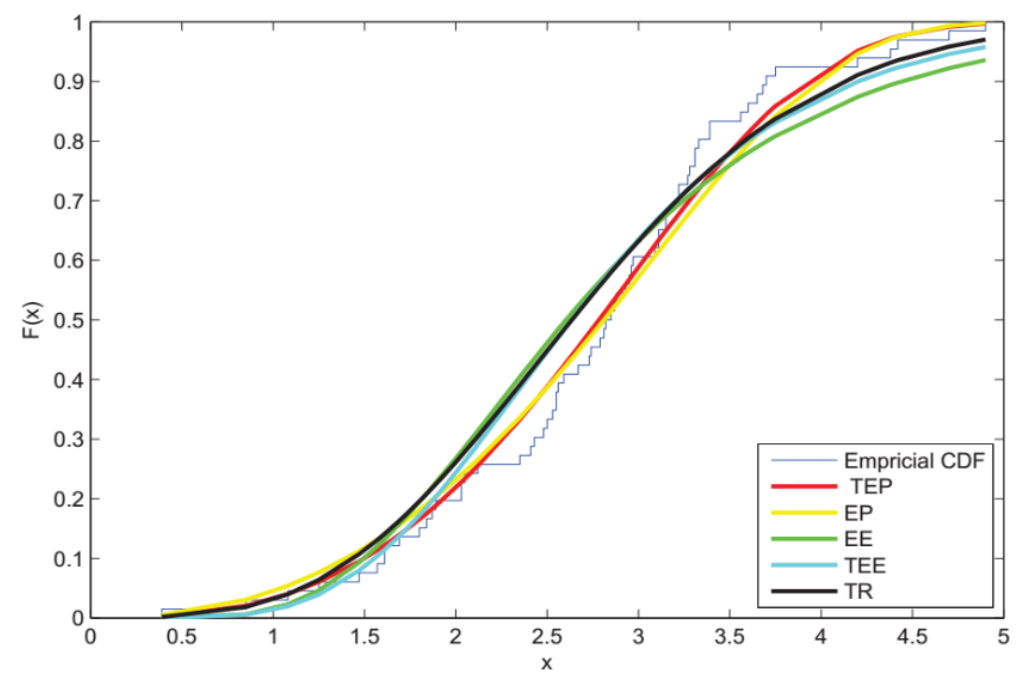

Figure 6. Empirical cdf and fitted cdfs for breaking stress data

\section{Conclusion}

In this study, we have proposed a new lifetime distribution which can be used as an alternative of EP distribution called as TEP. This new distribution having increasing, decrasing and bathtube hazard rate function has more flexibility than 


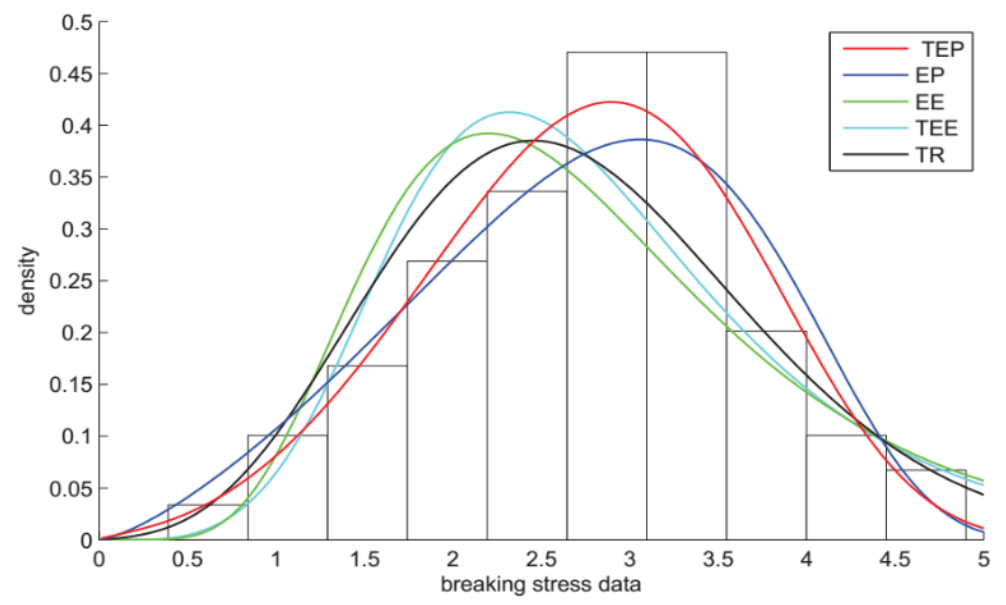

Figure 7. Fitted pdfs for breaking stress data

EP distribution. From two real data applications, it can have been concluded that TEP distribution has the best fitting among other fitted distributions. These real data applications show that TEP distribution is usefullness for modelling real data such as carbon fibres, operation and empirical data sets. These areas of application can be extended by using various real data sets which show fitting to TEP distribution.

\section{REFERENCES}

[1] Akdam, N., Kinaci, I., Saracoğlu, B., Statistical inference of stress-strength reliability for the exponential power (EP) distribution based on progressive type-II censored samples, Hacettepe Journal of Mathematics and Statistics, 46 (2017), 239-253.

[2] Al-Babtain, A., Fattah, A. A., Ahmed, A. H. N., Merovci, F., The Kumaraswamy-transmuted exponentiated modified Weibull distribution, Communications in Statistics-Simulation and Computation, 46 (2017), 3812-3832.

[3] Aryal, G. R., Tsokos, C. P., Transmuted Weibull distribution: A generalization of the Weibull probability distribution, European Journal of Pure and Applied Mathematics 4 (2011), 89102.

[4] Aryal, G. R., Tsokos, C. P., On the transmuted extreme value distribution with application, Nonlinear Analysis: Theory, Methods \& Applications, 71 (2009), 1401-1407.

[5] Ashour, S. K., Eltehiwy, M. A., Transmuted exponentiated Lomax distribution, Aust J Basic Appl Sci, 7 (2013), 658-667.

[6] Ashour, S. K., Eltehiwy, M. A., Transmuted lomax distribution, American Journal of Applied Mathematics and Statistics, 1 (2013), 121-127.

[7] Barriga, G. D., Louzada-Neto, F., Cancho, V. G. The complementary exponential power lifetime model, Computational statistics and data analysis, 55(3) (2011), 1250-1259.

[8] Chen Z. Statistical inference about the shape parameter of the exponential power distribution.Statistical Papers, 40(1) (1999), 459-468. 
[9] Dasgupta, R., On the distribution of burr with applications, Sankhya B, 73 (2011), 1-19.

[10] Elbatal, I., Aryal, G., On the Transmuted Additive Weibull Distribution, Austrian Journal of Statistics, 42 (2016), 117-132.

[11] El-Gohary, A., Alshamrani, A., Al-Otaibi, A. N., The generalized Gompertz distribution, Applied Mathematical Modelling, 37 (1-2) (2013), 13-24.

[12] Gupta, R. D., Kundu, D., Exponentiated exponential family: an alternative to gamma and Weibull distributions, Biometrical journal, 43 (2001), 117-130.

[13] Hussian, M. A., Transmuted exponentiated gamma distribution: A generalization of the exponentiated gamma probability distribution, Applied Mathematical Sciences, 8 (2014), 12971310.

[14] Khan, M S., King, R. and Hudson, I L., Transmuted Kumaraswamy Distribution. Statistics in Transition new series, 17 (2016), 183-210.

[15] Khan, M. S., King, R. and \& Hudson, I L., Transmuted Weibull distribution: Properties and estimation, Communications in Statistics-Theory and Methods, 46 (2017), 5394-5418.

[16] Khan, M. S., King, R. and Hudson, I L., Transmuted generalized Gompertz distribution with application, Journal of Statistical Theory and Applications, 16 (2017), 65-80.

[17] Mahmoud, M. R. and \& Mandouh, R M. On the transmuted Fréchet distribution, Journal of Applied Sciences Research, 9 (2013), 5553-5561.

[18] Merovci, F., Transmuted rayleigh distribution, Austrian Journal of Statistics, 42 (2013), 21-31.

[19] Merovci, F., Transmuted generalized Rayleigh distribution, Journal of Statistics Applications 83 Probability, 3 (2014), 9-20.

[20] Merovci, F., Transmuted lindley distribution, International Journal of Open Problems in Computer Science and Mathematics, 6 (2014), 63-72.

[21] Merovci, F., Transmuted exponentiated exponential distribution, Mathematical Sciences and Applications ENotes, 1 (2013) 112-122.

[22] Nichols, M. D., Padgett W. J., A bootstrap control chart for Weibull percentiles, Quality and Reliability Engineering International,, 22 (2006) 141-151.

[23] Smith, R.M., Bain, L.J., An exponential power life-testing distribution, Communications in Statistics, , 4(5) (1975), 469-481.

[24] Shahzad, M. N., Asghar, Z., Transmuted Dagum distribution: A more flexible and broad shaped hazard function model, Hacettepe Journal of Mathematics and Statistics, 45 (2016), $227-244$.

[25] Shaw, W. T. , Buckley, I. R., The Alchemy of Probability Distributions: Beyond Gram Charlier \& Cornish Fisher Expansions, and Skew-Normal or Kurtotic-Normal Distributions, Technical report, Financial Mathematics Group, King's College, London, U.K. 2007.

[26] Shaw, W. T., Buckley, I. R., The alchemy of probability distributions: beyond Gram-Charlier expansions, and a skewkurtotic-normal distribution from a rank transmutation map, arXiv preprint (2009) arXiv:0901.0434.

[27] Yousof, H. M., Alizadeh, M., Jahanshahi, S. M. A., Ramiresd, T. G., Ghoshe, I., Hamedani, G. G., The transmuted Topp-Leone G family of distributions: theory, characterizations and applications, Journal of Data Science, 15 (2017), 723-740. 